\title{
Histochemical Observations on Enlarged Submaxillary Glands Following Administration of Isoproterenol
}

\author{
Michio FukudA \\ Second Department of Oral Surgery, Osaka University Dental School, Osaka.
}

It has been reported that repeated administration of large doses of Isoproterenol Hydrochloride (ISO) caused an enlargement of the salivary glands of rats. Selye and Schneyer, have recently reported that repeated administration of Catecholamine, ISO, showed a marked increase in the weight of salivary glands. It was suggested that such increase resulted from a response to stimulation given to the sympathetic nerve system. The present paper deals with histological and histochemical observations of enlarged submaxillary glands of albino rats following administration of ISO.

\section{Materials and Methods}

ISO dissolved in distilled water was injected intraperitoneally to albino female rats of Sprague-Dawley strain, 80 days of age. The experiments were divided into the following two groups.

1. One consisted of five sub-groups administrated $10 \mathrm{mg}, 20 \mathrm{mg}, 40 \mathrm{mg}$, $80 \mathrm{mg}$ and $160 \mathrm{mg}$ of ISO, respectively, over a period of four days.

2. The other was administrated a daily dose $(16 \mathrm{mg})$ of ISO for five days. After cessation of administration, observations were made daily for seven days. The experimental and control rats were starved for the last $24 \mathrm{hrs}$., and were sacrificed by a blow on the head. The treated submaxillary glands were immediately dissected and frozen at $-70^{\circ} \mathrm{C}$. Fresh forzen sections of $12-18 \mu$ thick were made in a cryostat at $-20^{\circ} \mathrm{C}$ with a sliding microtome.

For the demonstration of the hydrolytic enzymes, alkaline phosphatase, acid phosphatase, non-specific esterase and $\beta$-glucuronidase, $10 \%$ formalin fixed cryostat sections were used. For the demonstration of oxidative enzymes, succinate dehydrogenase, NAD-dependent dehydrogenases (lactate, malate, glutamate, $\alpha$-glycerophosphate and $\beta$-hydroxybutyrate dehydrogenase) and monoamine oxidase, non-fixed cryostat sections were employed. Sudan black B and Sudan IV stain for lipids were applied in 10\% formalin fixed sections. Morel-Sisley Diazotization method for thyrosine, coupling tetrazonium method and alkali tetrazolium method for $\mathrm{SH}$ were also stained.

\section{Results}

Histopathological findings

Acinar cells of the submaxillary gland revealed a cytoplasmic enlargement and rare mitotic findings following a four day administration. Regarding a 
relationship between administration doses and an acinous enlargement, the large dose administrated groups showed a marked effect of enlargement, but the small dose groups showed no influence. Striated ducts and granular tubules revealed a gradual degeneration and they were compressed by enlarged acini. Especially, the granular tubules exhibited a marked reduction in number.

Recovery of submaxillary glands was observed after cessation of adminis. tration of ISO. Seven days after cessation of administration, recovery in cellular size of granular tubules and duct cells was distinct, and both cells showed almost the same size as normal cells but the acinar cells showed less size than normal acinar cells.

Histochemical findings

Alkaline phosphatase activity was confined to the enlarged myoepithelial cells and in blood vessles, but not to acini and ducts. Acid phosphatase reaction was positive in striated ducts and granular tubules and rather weak in the acini than in normal acini. The distribution pattern in the treated glands was almost normal. Esterase reaction was nearly the same as a reducing pattern of acid phosphatase in the treated animals. Moderate $\beta$-glucuronidase activity was found in striated ducts and granular tubules, and low activity in acini in normal glands. A staining pattern of the enlarged gland was essentially the same as that of normal animals. Succinic dehydrogenase activity, in the normal gland, was characterized by the presence of the highest activity in duct cells and moderate activity in granular tubules, and low activity in acini. In the administrated groups, the enzyme activity in granular tubules and ducts was not remarkably altered, but that in acini of the enlarged gland was decreased. In a succinic dehydrogenase staining of enlarged glands following large dose administration, duct systems with high stainability were significantly decreased in number, though duct cells exhibited a marked histological degeneration.

The distribution patterns in other dehydrogenases were similar to those of succinic dehydrogenase, but their enzymatic intensities were individually different.

Monoamine oxidase was moderately present in duct cells and granular tubules, and slightly in the acini of normal glands. The activity in the experimental gland was markedly stronger than the control gland in the acini.

The localization of proteins in the normal salivary glands was almost identical in three different staining methods employed, the proteins in ducts were stained more markedly than in acini. In enlarged glands, the stainability increased in ducts and acini.

Sudan black B and Sudan IV staining reactions were intense in ducts and slight in acini. Staining with Sudan IV was negligible in acini. The staining pattern of the enlarged gland was essentially the same as that of the control gland.

Histochemistry in recovering glands: Histochemical activities of alkaline phosphatase, acid phosphatase, esterase, $\beta$-glucuronidase and succinic dehydrogenase showed a trend similar to control glands. But the ISO treatment groups 
showed more marked staining than controls for proteins.

Monoamine oxidase reaction was not so potential as to assure recovery after cessation of 7 day's administration of ISO. Sudan black B and Sudan IV stains showed almost the same staining as that of the control gland.

\title{
Histochemical Observations on Epithelial and Mesenchymal Mucopolysaccharides: Histochemical Consideration of Histogenesis of the So-Called Mixed-Tumor of Minor Salivary Glands
}

\author{
Kinji Okamoto, Fusazo Taguchi*, Kazuo Sakamoto*, Haruo Hiraoka*, \\ Toshio YAGI, and Akitoshi Sugrmoto*
}

(Dept. of Oral Pathology, Osaka Univ. Dental School, Osaka,)

Two types of mucopolysaccharides (MPS) in animal tissue, epithelial and mesenchymal, have been demonstrated with histochemical methods by some authors in relation to the study on the so-called mixed tumor of the salivary gland. But the reported results are different according to the authors, and even the differentiating points between the two types are not always agreed. In the present study, MPS of normal and neoplastic tissues in 100 human cases and in 10 rats, including 38 human cases of the "mixed tumor" in minor salivary glands, are pursued histochemically in formol-fixed paraffin sections with the following methods: PAS-reaction ${ }^{1)}$, metachromatic reaction with toluidine blue (TB) ${ }^{2)}$ at $\mathrm{pH} 6.8,4.1$ and 2.3, alcian blue (AB) stain ${ }^{3}$, AB-PAS ("), aldehyde fuchsin (AF)-AB $\mathrm{B}^{5)}$, colloidal iron (CI) reaction ${ }^{6)}, \mathrm{CI}-\mathrm{PAS}^{6)}$, bi-col method ${ }^{7)}$ and digesting tests with hyaluronidase (Spurase) ${ }^{5)}$ and saliva ${ }^{2)}$.

The results obtained are summerized in Table I and II. There is no single characteristic reaction for each type of tissue MPS to assure a conclusive differentiation, because any reactions used are irregularly positive for both types of MPS. But combination of some reactions seems to be useful, and common findings for differentiation may be pointed out as follows :

1) PAS reaction is intensely positive for most epithelial mucoids, though weak or moderate for mesenchymal tissue.

2) Metachromasia with TB stain occurs in both of MPS, but in general epithelial reaction may be unstable and of ten negative at lower $\mathrm{pH}$, in contrast

\footnotetext{
* Present address : Dept. of Oral Pathology, Hiroshima Univerisity Dental School, Hiroshima.
} 